\title{
Neonatal epididymo-orchitis: An unusual manifestation of salmonellosis
}

Shaun Trecarten, Fadi Zu'bi, Mohammed Beaiti, Frank Papanikolaou

Division of Urology, The Hospital for Sick Children, Toronto, ON, Canada

Cite as: Can Urol Assoc J 2019 February 22; Epub ahead of print. http://dx.doi.org/10.5489/cuaj.5878

Published online February 22, 2019

$* * *$

\section{Introduction}

Epididymo-orchitis (EO) is a rare clinical presentation in the neonatal period. ${ }^{1}$ Previous cases have been reported in neonates in the context of bacterial infection with E. coli, ${ }^{2}$ Pseudomonas, ${ }^{3}$ and even Salmonella. ${ }^{4}$

Salmonellosis, either typhoidal or non-typhoidal, is usually spread by the feco-oral route. Typically presenting with an acute gastroenteritis, Salmonella has also been known to have extraintestinal manifestations, including osteomyelitis and reactive arthritis.

This paper describes a rare presentation of neonatal Salmonella EO and scrotal abscess, and is the first such case reported in Canada.

\section{Case presentation}

A 16 day old neonate presented to our institution with a 3 day history of right-sided scrotal swelling with progressive erythema and pain. He was persistently afebrile and tolerating his feeds, though he was passing 15-20 loose mustard-yellow stools daily. There were no sick close contacts and no recent travel. Peripheral blood and urine cultures were taken and he was started on intravenous ampicillin and tobramycin on admission. His C-reactive protein was 47, white cell count was 14.6 and urinalysis demonstrated trace red blood cells, but was negative for nitrites and leukocytes. A Doppler scrotal ultrasound scan identified features in keeping with right EO with a moderate to large hydrocele (Figure1). An abdominal ultrasound scan was carried out to rule out urinary tract abnormalities and it was reported as normal.

The blood cultures showed a significant growth of Salmonella javiana and the patient was started on IV cefotaxime. He underwent a lumbar puncture showing colourless CSF with a high white cell and protein count. A CSF culture was sent, which revealed no growth.

Despite the IV antibiotics, there was an increase in the right-sided hemiscrotal swelling and erythema (Figure 2). He underwent a repeat scrotal ultrasound demonstrating persistent EO and a new complex scrotal collection measuring 2 x 1.6 x $1.1 \mathrm{~cm}$ (Figure 3). He subsequently underwent a bedside incision and drainage of his abscess, and the pus swabs 
sent for culture were positive for light growth of Salmonella. He continued a total 2 week course of IV ceftazidime, and was switched to oral amoxicillin for another 12 days.

Approximately a month after he presented to hospital he was seen in the ambulatory Urology clinic where his parents reported a full recovery. On examination both testicles were palpable within the scrotum, and of normal size and consistency. The scrotal skin was not indurated and healed well postoperatively. His follow up ultrasound demonstrated resolution of the scrotal wall inflammation with no evidence of a persistent collection, and bilateral normal appearances of the epididymides and testicles.

\section{Discussion}

Our literature search revealed 6 other cases of neonatal Salmonella EO, only 2 of which were published after the year 2000.,5,6,7,8,9,10 This is the first such case to be reported in Canada, and the first linked to the Salmonella javiana serotype, which is rarely associated with the development of abscesses or found in blood/urine cultures. ${ }^{11}$

Patients less than 3 months of age, such as the presented case, are thought to be at a higher risk of Salmonella bacteremia due to decreased gut associated lymphoid tissue, decreased gastric acidity and rapid gastric emptying. 4, 6. 12 Other risk factors include asplenia and immunocompromised states. Hematogenous seeding by a Salmonella bacteremia is currently the preferred mechanism of infection, rather than ascending infection via the urinary tract. ${ }^{6,7}$ The sequence of Salmonella EO as a consequence of disseminated infection seems to be most likely, as similarly to our case, urine cultures have historically been negative. While the exact reason for dissemination specifically to the scrotum is unknown, there are some studies showing an association between Salmonella strains causing extraintestinal disease and those carrying an additional genetic locus called $s p v$, which enhances the virulence of the bacteria. ${ }^{13}$

There is clinical consensus that in the presence of a positive blood culture for Salmonella, a lumbar puncture should be performed for cerebrospinal fluid (CSF) culture to rule out meningitis. ${ }^{12}$ Similar to our case, a lumbar puncture was performed in both case reports with positive blood cultures. While the blood culture is not always positive, as was the case in 5 of the 7 reported cases, clinicians should always treat those with confirmed Salmonella EO as having had a bacteremia, and consult Infectious Diseases about further investigation. $^{12}$

Surgery was ultimately required in all of the reported cases of Salmonella EO. Whether the case was initially thought to be a strangulated inguinal hernia (and intraoperatively found to be an abscess) or an abscess was identified preoperatively, an incision and drainage was required despite a trial of intravenous antibiotics. Thereafter, patients seem to make a good recovery. Accordingly, we recommend early involvement of Urological surgeons in all cases of Salmonella EO.

Salmonella infection poses an important public health concern and is a notifiable disease in Canada. Common sources of non-typhoidal disease include animal contact (especially reptiles), and contaminated foods including poultry, eggs, dairy, ground beef and produce. To help isolate the source of infection, parents should be asked about any domestic 
animals at home, any sick close contacts, or exposure to animal treats which commonly contain raw meat. ${ }^{12}$

The Canadian Pediatric Society has published recent guidelines on Salmonella infections in the pediatric population. ${ }^{12}$ While the recommendation for empiric antibiotic treatment initially is ceftriaxone, our patient was commenced on ceftazidime due to our reported sensitivities. ${ }^{12}$ Stepping down to oral therapy should be based on antimicrobial sensitivity. The total duration of oral antibiotic therapy remains controversial. ${ }^{12}$

Salmonella EO is a rare entity but an important manifestation of disseminated Salmonella infection. There should be close multidisciplinary involvement of Urological surgeons (as future abscess is possible), Infectious Disease consultants (to help guide investigation of disseminated infection and selection/duration of antibiotic treatment), and Public Health (to help isolate the source and prevent further spread).

\section{Learning points}

- Risk factors for pediatric Salmonellosis include: age of patient is less than 3 months, asplenic state and immunosuppression.

- In the presence of disseminated disease or positive blood cultures for Salmonella, the Canadian Pediatric Society recommends empiric IV ceftriaxone.

- Patients with Salmonella EO are likely to progress to require incision and drainage of abscess to fully recover. 


\section{References}

1. Chiang M-C, Chen H-W, Fu R-H, et al. Clinical features of testicular torsion and epididymo-orchitis in infants younger than 3 months. J Pediatr Surg 2007;42:1574-7.

2. Fromme HB, Barrett Fromme H, Msezane L, et al. Acute Scrotal Swelling in a Newborn With Bacteremia. Clin Pediatr 2008;47:827-8.

3. Kabiri M, Barkat A, El Ajaje H, et al. Neonatal epididymo-orchitis caused by Pseudomonas aeruginosa: a case report. Cases $J$ 2010;3:44.

4. Ngoo A, Rodriguez-Acevedo O, Carroll D, et al. Salmonella epididymo-orchitis presenting as acute scrotum in a 2-week-old infant. J Paediatr Child Health [Internet] 2018 November [cited 2019 Jan 13] Available from: http://dx.doi.org/10.1111/jpc.14295. Accessed 2019 Jan 13.

5. Foster R, Weber TR, Kleiman M, et al. Salmonella Enteritidis: Testicular Abscess in a Newborn. J Urol 1983;130:790-1.

6. Feder HM, Zempsky W. Neonatal Salmonella Orchitis. Infect Dis Clin Pract 2005;13:313-4.

7. Huang CB, Chuang JH. Acute scrotal inflammation caused by Salmonella in young infants. Pediatr Infect Dis J 1997;16:1091-2.

8. Berner R, Schumacher RF, Zimmerhackl LB, et al. Salmonella enteritidis orchitis in a 10-week-old boy. Acta Paediatr 1994;83:992-3.

9. Hakim A, Bradley H. Salmonella epididymo-orchitis in infancy and childhood. Clin Pediatr 1992;31:120-2.

10. Uwyyed K, Korman SH, Bar-Oz B, et al. Scrotal abscess with bacteremia caused by Salmonella group D after ritual circumcision. Pediatr Infect Dis J 1990;9:65-6.

11. Centers for Disease Control and Prevention (CDC). An Atlas of Salmonella in the United States, 1968-201. Laboratory-based Enteric Disease Surveillance [Internet]. Atlanta, Georgia: US Department of Health and Human Services, CDC, 2013 [updated 2013, cited 2019 Feb 19]. Available from: https://www.cdc.gov/salmonella/pdf/javiana-508c.pdf. Accessed 2019 Feb 19

12. Robinson JL. Salmonella infections in Canadian children [Internet]. Canadian Pediatric Society. 2018 [updated 2018 Dec 03, cited 2019 Jan 13]. Available from: https://www.cps.ca/en/documents/position/salmonella-infections-in-canadianchildren. Accesseed 2019 Jan 13.

13. Guiney DG, Fierer J. The Role of the spv Genes in Salmonella Pathogenesis. Front Microbiol. 2011 Jun 14;2:129. 


\section{Figures and Tables}

Fig. 1. Initial scrotal ultrasound demonstrates heterogeneous echogenicity and hyperemia of the right testicle. The epididymis is echogenic and hyperemic. A moderate to large hydrocele is present with internal echoes. The scrotal wall is markedly thickened.
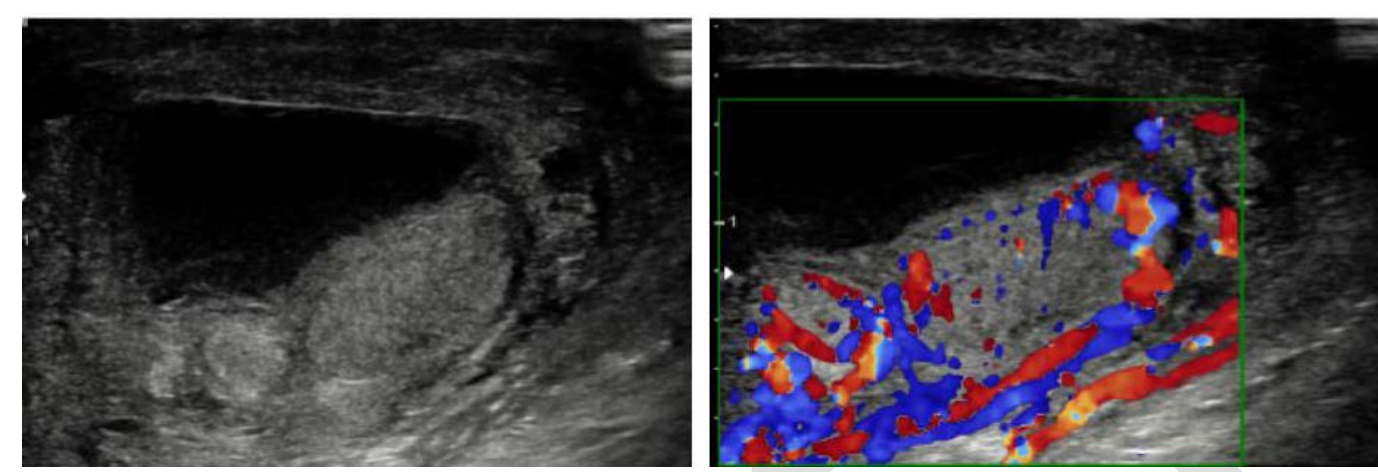

Fig. 2. Right-sided hemiscrotal swelling and erythema.

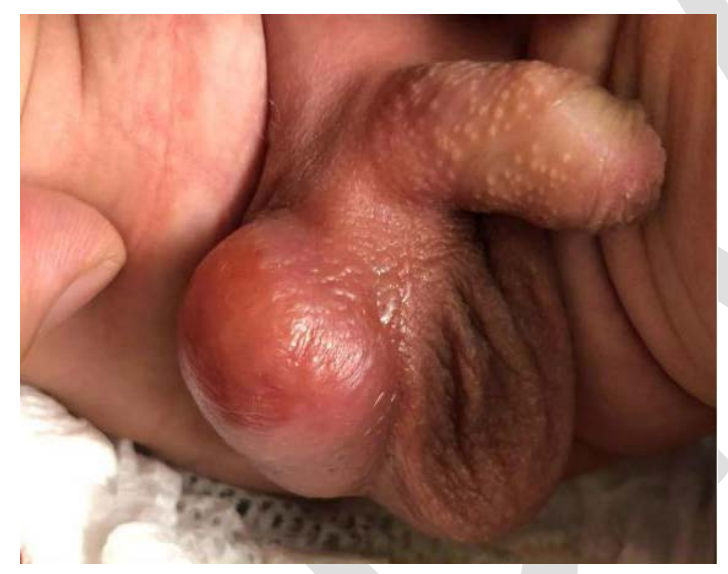


Fig. 3. Scrotal ultrasound demonstrates a right-sided moderate to large hydrocele with internal echoes. There is also marked thickening and hyperemia of the wall of the right side of the scrotum with a complex collection measuring $2.0 \times 1.6 \times 1.1 \mathrm{~cm}$.
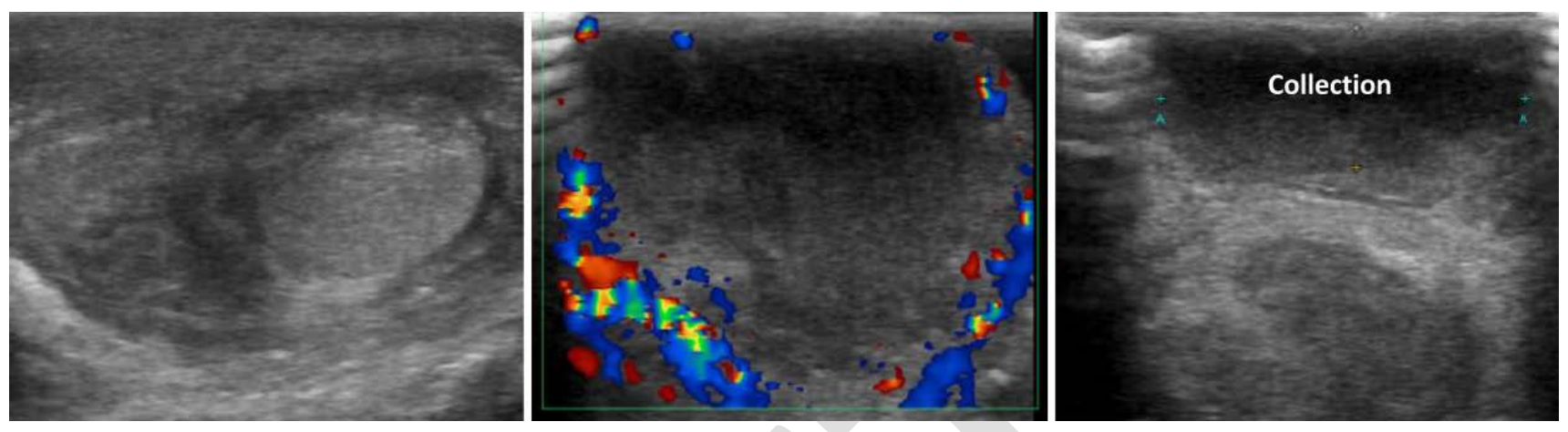